\title{
Identificação de espécies de Malassezia em pacientes com pitiríase versicolor em Goiânia-Go
}

\author{
Identification of Malassezia species in patients with \\ pityriasis versicolor in Goiânia-G0
}

\section{Karla Carvalho Miranda ${ }^{1}$, Crystiane Rodrigues de Araujo ${ }^{1}$, Ailton José Soares ${ }^{1}$, Janine de Aquino Lemos ${ }^{1}$, Lúcia Kioko Hasimoto e Souza ${ }^{1}$ e Maria do Rosário Rodrigues Silva ${ }^{1}$}

\section{RESUMO}

O objetivo deste estudo foi verificar a freqüência de pitiriase versicolor e identificar leveduras do gênero Malassezia, de pacientes encaminhados ao laboratório de Micologia da Universidade Federal de Goiás em Goiânia. Foram diagnosticados 95 casos de pitíriase versicolor e identificados quatro espécies de Malassezia: Malassezia furfur, Malassezia sympodialis, Malassezia globosa e Malassezia obtusa.

Palavras-chaves: Pitiríase versicolor. Malassezia spp. Malassezia furfur.

\section{ABSTRACT}

The objectives of this study were to investigate the frequency of pityriasis versicolor occurrence and to identify yeasts of the genus Malassezia in patients at the mycology laboratory of the Federal University of Goias, in Goiânia, State of Goiás. Ninety-five cases of pityriasis versicolor were diagnosed, and four species of Malassezia were identified: Malassezia furfur, Malassezia sympodialis, Malassezia globosa and Malassezia obtusa.

Key-words: Pityriasis versicolor. Malassezia spp. Malassezia furfur.

As leveduras do gênero Malasseziapodem produzir diferentes tipos de infecções cutâneas superficiais como pitiríase versicolor, dermatite seborreica, foliculites, dermatite atópica e eventualmente pode ocorrer disseminaçã $0^{578}$. 0 gênero Malassezia tem sido englobado em 11 espécies distintas: Malassezia globosa, M. obtusa, M. restricta, M. slooffiae, M. furfur, M. sympodialis (lipodependentes), e M. pachydermatis (não lipodependente) M. dermatis, M. nana, M. japonica e M. yamatoensis ${ }^{412}$.

Amostras de pele foram coletadas de 842 pacientes portadores de infecções fúngicas superficiais encaminhados ao Laboratório de Micologia, durante 0 ano de 2004, para caracterização e identificação de fungos do gênero Malassezia. Estas leveduras foram previamente identificadas, através de exame direto usando $\mathrm{KOH}$ a $40 \%$, e cultivadas em meios de ágar Dixon modificado ajustado em pH 6,0 e Ágar Sabouraud dextrose suplementado com cloranfenicol $(0.05 \%)$ e incubadas a $32^{\circ} \mathrm{C}$, por até 15 dias. Amostras positivas ao exame direto, mas que não cresceram em meio de cultura, foram recultivadas até a obtenção de crescimento. A identificação dos isolados foi feita segundo a técnica descrita por Guého cols $1996^{4}$.
Ao exame direto, leveduras brotantes e hifas curtas e tortuosas características de Malassezia sp foram visualizadas em 95 amostras.

Pela diferença de comportamento, na assimilação dos tweens 20, 40, 60 e 80, e pelas características morfológicas exibidas, na coloração de Gram, foi possível identificar $77,8 \%$ de $M$. furfur, 11,4\% de M. sympodialis, 8,4\% de M. obtusa e 2,1\% de M. globosa (Tabela 1 ).

Tabela 1 - Caracterização de 95 espécies de Malassezia sp de pacientes com pitiríase versicolor segundo o local da lesão.

\begin{tabular}{|c|c|c|c|c|c|c|c|c|c|c|}
\hline \multirow[b]{3}{*}{ Local da lesão } & \multicolumn{8}{|c|}{ Espécies de Malassezia } & & \\
\hline & \multicolumn{2}{|c|}{ furfur } & \multicolumn{2}{|c|}{ sympodialis } & \multicolumn{2}{|c|}{ obtusa } & \multicolumn{2}{|c|}{ globosa } & \multicolumn{2}{|c|}{ Total } \\
\hline & $\mathrm{n}^{0}$ & $\%$ & $\mathrm{n}^{-0}$ & $\%$ & $\overline{\mathrm{n}^{0}}$ & $\%$ & $\mathrm{n}^{0}$ & $\%$ & $\mathrm{n}^{0}$ & $\%$ \\
\hline Face & 2 & 2,0 & 1 & 1,0 & 1 & 1,0 & - & & 4 & 4,2 \\
\hline Pescoço & 7 & 7,4 & - & & - & & - & & 7 & 7,4 \\
\hline Braços & 8 & 8,4 & 1 & 1,0 & 1 & 1,0 & - & & 10 & 10,5 \\
\hline Tronco & 27 & 28,4 & 4 & 4,2 & 1 & 1,0 & 1 & 1,0 & 33 & 34,7 \\
\hline Abdomen & 5 & 5,3 & 1 & 1,0 & - & & - & & 6 & 6,3 \\
\hline Dorso & 21 & 22,1 & 4 & 4,2 & 5 & 5,3 & 1 & 1,0 & 31 & 32,6 \\
\hline Coxas & 4 & 4,2 & - & & - & & - & & 4 & 4,2 \\
\hline Total & 74 & 77,8 & 11 & 11,4 & 8 & 8,3 & 2 & 2,0 & 95 & \\
\hline
\end{tabular}

\footnotetext{
1. Laboratório de Micologia do Instituto de Patologia Tropical e Saúde Pública da Universidade Federal de Goiás, Goiânia, G0.

Endereço para correspondência: Prof ${ }^{a}$ Maria do Rosário Rodrigues Silva. Rua 15 n $^{\circ} 108$ Apt $^{\circ}$ 700, Setor 0este, 74140-090 Goiânia, G0.

Tel: $55623209-6127$

e-mail: rosario@iptsp.ufg.br

Recebido para publicação em 22/11/2005

Aceito em 4/12/2006
} 
Lesões causadas por Malassezia sp foram encontradas, predominantemente, no tronco e dorso, com uma porcentagem de $34,7 \%$ e $32,6 \%$, respectivamente. 0 agente etiológico mais isolado nestes locais foi $M$. furfur, responsável por 50,5\% (48/95) dos casos (Tabela 1).

Dentre as infecções causadas por leveduras do gênero Malassezia, destaca-se a pitiríase versicolor que se mostra bastante comum em adolescentes jovens, sendo considerada de distribuição mundial ${ }^{6}$. A identificação de 95 (11,3\%) casos de pitiríase versicolor observada de um total de 842 amostras de lesões superficiais, mostra que esta micose tem uma elevada frequiência em nosso meio. Porcentagens semelhantes às encontradas em nosso trabalho, foram verificadas na Turquia e na Nigéria ${ }^{39}$.

Neste estudo, foi verificado que pitiríase versicolor ocorreu com maior freqüência em adultos jovens (11-30 anos), do sexo feminino (66,3\%), com localização no tronco em 34,7\%. Em 62 pacientes com pitiríase versicolor oriundos da Tunísia ${ }^{10}$, a faixa etária de 15 a 30 anos ocorreu em $65 \%$ dos casos. Na República Dominicana, de 100 pacientes analisados, 61\% estavam na faixa de 11 a 30 anos, com predomínio do sexo feminino (62\%) e com lesões freqüentemente localizadas no tronco $(70 \%)^{1}$. A maior colonização no tronco e dorso pode ser explicada pela elevada densidade de glândulas sebáceas nesta área, o que preenche o requerimento de ácidos graxos exigidos pelas leveduras lipofílicas como $M$. furfur ${ }^{10}$.

As quatro espécies de Malassezia lipodependentes identificadas, atuam como agentes de pitiríase versicolor. M. furfur foi isolada em $77,8 \%$ o que mostra que esta espécie continua predominante em nosso meio. Embora até a década de 1990, Malassezia furfur tenha sido o principal agente de pitiríase versicolor ${ }^{11}$, nos últimos anos $M$. globosa encontrase como o agente mais comumente diagnosticado. Nesta casuística, este último agente foi isolado em apenas duas amostras. Malassezia sympodialis, espécie encontrada em $11,4 \%$ dos casos, foi também identificada com relativa frequiência na Espanha, onde foi observada uma porcentagem de 40,5 \% em 79 casos estudados ${ }^{2}$. Em conclusão, lesões de tronco e dorso dos adultos jovens, do sexo feminino, predominaram em nossa casuística, sendo $M$. furfur o agente etiológico mais comumente associado à pitiríase versicolor, evidenciando a patogenicidade dessa espécie.

\section{REFERÊNCIAS BIBLIOGRÁFICAS}

1. Arenas R, Isa RI, Cruz AC. Pitiriasis Versicolor em Santo Domingo, República Dominicana. Datos morfológicos de Malassezia spp. In vivo em 100 casos. Revista Iberoamericana de Micologia 18:29-32, 2001.

2. Aspiroz C, Ara M, Varea M, Rezusta A \& Rubio C. Isolation of Malassezia globosa and $M$. sympodialis from patients with pityriasis versicolor in Spain. Mycopathologia 154:111-117, 2002.

3. Çelik E, Ilkit M, Tanir F. Prevalence and causative agents of superficial mycoses in a textile factory in Adana, Turkey. Mycoses 46:311-315, 2003.

4. Guého E, Midgley G, Guillot J. The genus Malassezia with description of four new species. Antonie van Leeuwnhock 69:337-355, 1996.

5. Gupta AK, Kohli Y. Prevalence of Malassezia species on various body sites in clinically healthy subjects representing different age groups. Medical Mycology 42:35-42, 2004.

6. Ingordo V, Naldi L, Colecchias B, Licci N. Prevalence of pityriasis versicolor in young Italian sailors. British Journal of Dermatology 149:1270-1272, 2003.

7. Ljubojevic S, Skerlev M, Lipozencic J, Juzbasic AB. The role of Malassezia furfur in dermatology. Clinics in Dermatology 20:179-182, 2002.

8. Midgley G. The lipophilic yeast: state of the art and prospects. Medical Mycology 6:103-110, 2000.

9. Ogunbiyi A0, Owoaje E, Ndahi A. Prevalence of skin disorders in school children in Ibadan, Nigeria. Pediatric Dermatology 22:1-6, 2005.

10. Salah S Ben, Makni F, Marrakchi S, Sellami H, Cheikhrouhou, Bouassida S, Zahaf A, Ayadi A. Identification of Malassezia species from Tunisian patients with pityriasis versicolor and normal subjects. Mycoses 48:242-245, 2005.

11. Senczeck D, Siesenop U, Bohm KH. Characterization of Malassezia species by means of phenotypic characteristics and detection of electrophoretic karyotypes by pulsed- field gel electrophoresis (PFGE). Mycoses 42:409414, 1999.

12. Sugita T, Tajima M, Takashima M, Amaya M, Saito M, Tsuboi R, Nishikawa A. A new yeast, Malassezia yamatoensis, isolated from a patient with seborrheic dermatitis, and its distribution in patients and healthy subjects. Microbiolology and Immunology 48:579-583, 2004. 